\title{
Evaluation of fluorescent in-situ hybridization technique for diagnosis of malaria in Ahero Sub-County hospital, Kenya
}

Regina Kandie ${ }^{1,2^{*}}$, Rachel Ochola ${ }^{3}$ and Kariuki Njaanake ${ }^{1}$

\begin{abstract}
Background: Malaria is a major cause of morbidity and mortality. Treatment of malaria in a timely manner could avert deaths. Treatment ultimately relies on the rapid and accurate diagnosis. Fluorescence in situ hybridization (FISH), a cytogenetic technique based on detection of specific nucleic acid, has the potential to address the limitations of the current diagnostic approaches. This study investigates further the performance of FISH for the diagnosis of malaria in a rural setting in Western Kenya.

Methods: Blood samples from 302 patients presenting with fever (temperature $\geq 37.5^{\circ} \mathrm{C}$ ) were examined for malaria using the Giemsa microscopy (GM), rapid diagnostic test (RDT), polymerase chain reaction (PCR) and FISH.

Results: The sensitivity and specificity of FISH was $85.6 \%$ and $96.2 \%$ respectively, while the corresponding values for GM were $82.2 \%$ and $100 \%$ respectively. RDT and PCR had sensitivities of $91.1 \%$ and $98.9 \%$, respectively with their specificities being 89.6 and 100\%, respectively. The positive predictive values for RDT, GM, FISH and PCR were 78. $8 \%, 100 \%, 90.6 \%$ and 100\%, respectively. The negative predictive values for RDT, GM, FISH and PCR were 96.0\%, 93. $0 \%, 94.0 \%$ and $99.5 \%$, respectively. Their respective diagnostic accuracies were 90.1\%, 94.7\% 93.0\% and 99.7\%.

Conclusion: The present study demonstrates that the specificity and reproducibility of FISH assays are high, thus adding to the growing evidence on the potential of the technique as an effective tool for the detection of malaria parasites in remote settings.
\end{abstract}

Keywords: Malaria, Fluorescent in-situ hybridization, Microscopy, Rapid diagnostic tests, Sensitivity, Specificity

\section{Background}

Malaria is one of the most important public health problems worldwide, and a leading cause of morbidity and mortality in many developing tropical countries, with young children and pregnant women being the most affected groups within populations [1]. An estimated 3.4 billion people in 106 countries and territories live in high malaria risk areas [2]. Worldwide, a child dies of malaria every 2 min which translates to about 3000 children every day [3]. About $88 \%$ of all malaria cases in 2015 are estimated to

\footnotetext{
* Correspondence: regkandie@yahoo.com

${ }^{1}$ Department of Medical Microbiology, College of Health Sciences, University of Nairobi, Nairobi, Kenya

${ }^{2}$ National Malaria Control Program, Ministry of Health, Nairobi, Kenya

Full list of author information is available at the end of the article
}

have occurred in sub-Saharan Africa where it is still the main cause of death and a major threat to child health, followed by the World Health Organization (WHO) South-East Asia Region (7\%) and the WHO Eastern Mediterranean Region (2\%). During the same year, it was estimated that most (92\%) malaria-related deaths were in the WHO African Region, followed by the WHO South-East Asia Region (6\%) and the WHO Eastern Mediterranean Region (2\%) [4]. Malaria is mainly concentrated in low and middle-income countries where it mostly affects the poorest and marginalized communities. Such communities have the highest risks associated with malaria, and the least access to effective services for prevention, diagnosis and treatment $[4,5]$. 
In Kenya, about $52 \%$ of the population is at risk of contracting malaria [6]. The goal of the National Malaria Strategy 2009-2017 is to reduce morbidity and mortality associated with malaria by $30 \%$ by the year 2009 and to maintain it through 2015 [7]. Countrywide, prevalence of malaria has declined from $11 \%$ to $8 \%$ between 2010 and 2015. The endemic region along Lake Victoria has the highest malaria prevalence $(27 \%)[7,8]$.

Following WHO's recommendation that all cases of suspected malaria be confirmed using parasite-based diagnostic testing, either microscopy or rapid diagnostic test (RDT) before commencement of treatment, there has been an impressive increase in the proportion of suspected malaria cases receiving a diagnostic test since 2010, especially in the African Region [8]. However, malaria diagnosis through microscopy has been shown to have low sensitivity and specificity, is laborious and requires highly trained personnel which negatively affects health outcomes and optimal use of resources [9-11].

Malaria RDTs provide a valuable tool especially in field studies as they are able to counter the inadequacies of other diagnostic approaches. In countries where malaria is endemic, they provide an alternative approach in settings where microscopy is not feasible [12]. Moreover, they can be used to complement microscopy as they provide timely results and in settings where microscopy experience is limited as is the case in most non-endemic countries [12, 13]. The method is based on the immunochromatographic principles, that is, chromatography coupled with monoclonal antibodies that capture antigens of the parasite including pan-lactate dehydrogenase (pLDH) or histidine-rich protein-2 (HRP-2) [14]. As with microscopy, RDTs have limited detection threshold, particularly in situations where parasitaemia is low [14, 15]. Moreover, despite its wide use, the RDT method based on serum antibody is also not specific $[16,17]$.

Currently, polymerase chain reaction (PCR) assay is used chiefly for research purposes or for speciation in reference laboratories. This method involves detecting circulating malaria parasites by detecting parasite DNA through amplification of ribosomal RNA genes. This can detect parasites at low parasitaemia, even below 5 parasites/ $\mu$ l of blood, for all five human Plasmodium spp. [18]. However, molecular techniques are expensive and require specialized laboratory facilities and highly trained personnel, owing to the related cost of labor and difficulties in accessing the reagents, compared to the examination of blood smears [19]. These drawbacks present an important impediment to its adoption in malaria reference laboratories located in endemic resource-poor regions of the world.

In Kenya, Giemsa microscopy (GM) is traditionally the gold standard for diagnosis. Under optimum conditions, microscopy can detect $20-50$ parasites per $\mu \mathrm{L}$ blood, but such sensitivity is rarely achieved in routine diagnosis. On the other hand, parasite nucleic acids can be detected using PCR. Although this technique may be slightly more sensitive than smear microscopy, it is of limited utility for the diagnosis of acutely ill patients in the standard healthcare setting due to its unavailability and long turnaround time to results. PCR results are often not available quickly enough to be of value in establishing the diagnosis of malaria in such patients. Other alternative laboratory methods for the detection of malaria parasites including the quantitative buffy-coat centrifugal haematology system, immunofluorescence and ELISA tests for the detection of $P$. falciparum antigen have been recently developed. None of these tests however, are used routinely as they are far too complicated or expensive for use in routine diagnosis especially in a developing countries including Kenya. There is therefore a need for sensitive, easily accessible and affordable diagnostic tools to facilitate targeted treatment and management of malaria. In addition, new diagnostics that can detect low levels of parasitaemia in asymptomatic individuals and, in the case of $P$. vivax, in the blood of asymptomatic individuals who may serve as reservoirs for mosquito infections, are much needed [20].

One of the promising molecular techniques in malaria diagnosis is the fluorescent in-situ hybridization (FISH) assay. This is a cytogenetic technique that is able to detect and localize the specific nucleic acid (DNA or RNA) sequences by hybridizing with complementary sequences labeled with fluorescent probes. The assay gives results within 45 mins and can circumvent the drawbacks characterising PCR such as requirement of sophiticated equipment, highly trained personnel and prolonged time to results. Specific FISH assays that can be deployed in peripheral laboratories of limited resource nations where poverty-related diseases including malaria and tuberculosis are endemic, have been developed [21, 22]. There is accumulating evidence that FISH assay technique can be a valuable tool for the diagnosis of malaria under routine clinical settings. Indeed, a recent study done in a malaria holoendemic region of Kenya showed that P-Genus FISH assay has great potential in detecting low density parasites [23]. This study aimed at generating additional evidence on use of FISH in a clinical setting by evaluating the performance of the technique in malaria diagnosis in a rural malaria-endemic community in Western Kenya.

\section{Methods}

\section{Study area}

The study was conducted in Ahero sub-County Hospital, Kisumu County of former Nyanza province, Western Kenya. The County covers an area of $2085.9 \mathrm{~km}^{2}$ and has a population of 968,909 [20]. This area is situated in the lake region malaria epidemiological zone. The massive vegetation cover, swampy low altitude and consistent 
rainfall make the region highly conducive for mosquito breeding and high malaria transmissions.

\section{Study design}

This was a descriptive cross-sectional study in which blood samples were collected from patients presenting with fever of $\geq 37.5{ }^{\circ} \mathrm{C}$ and examined for malaria parasites using rapid diagnostic test (RDT) kit, Giemsa microscopy $(\mathrm{GM})$, polymerase chain reaction (PCR) and fluorescent in-situ hybridization (FISH).

\section{Sample size}

The minimum required sample size for the study was calculated using the formula by Lwanga and Lemeshow [24].

$$
n=z_{\propto}^{2} \frac{p(1-p)}{d^{2}}
$$

Where;

$n$ is the sample size

$z_{\propto / 2}$ is the normal standard deviate for a given level of significance $\left(5 \%, z_{\propto / 2}=1.962\right)$

$p$ is the prevalence of infection expressed as a proportion

$d$ is the desired level of precision (0.05).

Malaria prevalence around Lake Victoria is estimated to be $26.7 \%$ [25]. Using $5 \%$ as the precision level and level of significance, this gave a minimum sample size (n) of 302 patients.

\section{Sampling procedure}

Patients visiting the outpatient clinics of Ahero subCounty Hospital in October 2016 and presenting with fever $\left(\geq 37.5^{\circ} \mathrm{C}\right)$ and with suspected malaria were enrolled sequentially into the study after informed consent was obtained. Exclusion criteria were lack of consent from the caregivers and symptoms associated with signs of severe malaria as defined by WHO [26]. Also excluded were those with had been on anti-malarial drugs within the previous 7 days.

\section{Laboratory procedures: Collection and examination of blood samples}

From each enrolled subject, $2 \mathrm{ml}$ of venous blood sample was drawn and examined for the presence of malaria parasites using the GM, RDT, PCR and FISH.

\section{Giemsa microscopy}

Giemsa microscopy was performed as outlined in the WHO guidelines [27]. Blood films (thin and thick) were prepared and then stained using $10 \%$ Giemsa stain. The slides were examined microscopically using $\times 1000$ magnification. Thick blood film were examined for the presence of parasites and density while species confirmation was performed on thin film. Quantification of malaria parasites was done for positive slides by counting the parasites against 200 white blood cells. Samples were classified as negative if no malaria parasites were observed following examination of 100 microscopic fields. The readings were done by two independent microscopist who were blinded. In cases where the results were discordant, the readings from a third microscopist served as a tie breaker.

\section{Rapid diagnostic tests}

Blood samples were tested for malaria parasite antigen using SD Bioline Malaria Ag P.f/Pan (Standard Diagnostics Inc., Korea), RDT kits as outlined in the manufacturers' instructions inserts.

\section{Fluorescence in situ hybridization assays}

The FISH assays were conducted on all the samples as per the manufacturer's instructions supplied as part of the P. falciparum/P. vivax FISH combo and Plasmodium Genus FISH Kit (ID-FISH Technology Inc., Palo Alto, CA, USA). Briefly, thin smears were prepared from EDTA whole blood mixed with smear preparation reagent (SPR) (3 parts blood: 1 part SPR by volume). Each smear was prepared from $4 \mu \mathrm{l}$ of the mixture, dried in the air and fixed in methanol. Hybridization buffer with probe mix $(12 \mu \mathrm{l})$ was added to each fixed smear. The smear was covered with a cover slip and placed in a humid chamber at $37{ }^{\circ} \mathrm{C}$ for $30 \mathrm{~min}$ to allow for hybridization to occur. The smear was then washed with wash buffer twice, rinsed with rinse buffer and dried in the dark. A drop of plasmodium counterstain was added to the dry smear. The smear was then covered with a cover slip and examined using a fluorescence microscope (magnification: $x$ 1000 ) fitted with a green filter (excitation and emission of $560 \mathrm{~nm}$ and $630 \mathrm{~nm}$ respectively).

\section{Polymerase chain reaction}

Briefly, PCR analyses were performed on purified DNA using primers (Forward 5' - GCTCTTTCTTGATTTCTT GGATG -3' and Reverse 5'AGCAGGTTAAGATCTCG TTCG-3') for plasmodium genus using Rotor gene $Q$ real time PCR cycler.

\section{Data management and statistical analysis}

Data were entered in Microsoft Excel spread sheet and imported into Stata 13.0 for statistical analysis. Performance of the diagnostic methods was assessed with the combination of PCR and GM results serving as the gold standard for malaria detection. All but one sample which were positive by GM were also positive by PCR. The sample was taken as part of the reference standard. Demographic attributes of the study participants were summarized using appropriate descriptive statistics. The number of true positives (TP), true negatives $(\mathrm{TN})$, false 
positives (FP) and false negatives (FN) were computed. Sensitivity was calculated as TP/ $(\mathrm{TP}+\mathrm{FN})$. Specificity was calculated as $\mathrm{TN} /(\mathrm{TN}+\mathrm{FP})$. The positive predictive value (PPV) was calculated as $\mathrm{TP} /(\mathrm{TP}+\mathrm{FP})$ and negative predictive value (NPV) as TN/ (FN + TN). The accuracy of the test, defined as proportion of all tests that gave a correct result, was calculated as $(\mathrm{TP}+\mathrm{TN}) /$ number of all tests. Variations in proportions of outcomes based on different diagnostic techniques were tested using chi square ( $\chi 2)$ test. For all statistical tests, the threshold for statistical significance was set at a $p$-value of less than 0.05 .

The density of malaria parasite was determined as follows [28]:

$$
\text { Number of parasites } / \mu \text { l blood }=\frac{\text { Number of parasites observed } x 8000}{\text { Number of white blood cells observed }}
$$

Cohen's $\mathrm{k}$ was used to determine if there was agreement between FISH, Giemsa-microscopy, microscopy and/or PCR and RDT in the diagnoses of malaria.

\section{Results}

Demographic characteristics of the study participants A total of 302 children were enrolled in the study with the majority being females (65.6\%). Their ages ranged from one to 79 months with the median (interquartile range) age being 21 (9-33) months. Children less than 6 months of age constituted 18.2\%; between 6 and 24 months, 40.4\%; between 25 and 59 months, 39.7\%; and $>60$ months, $1.7 \%$ of the study participants.

\section{Prevalence of malaria}

The prevalence of infections with malaria parasites among the samples tested using the four diagnostic techniques under study is shown in Table 1. Overall, 81 (28.1\%) and 104 (34.4\%) samples were positive for malaria parasites when tested using FISH and RDTs, respectively. Eight nine samples (29.5\%) were positive for malaria parasites based on PCR results only. Ninety samples (29.8\%) were positive for malaria parasites by microscopy and/or PCR.

\section{Evaluation of the performance of the diagnostic tests}

The sensitivity, specificity, positive predictive value (PPV), and negative predictive value (NPV) of the tests are shown

Table 1 Summary of Plasmodium Genus results based on diagnostic tests utilized in the study

\begin{tabular}{llllll}
\hline Test & \multicolumn{2}{l}{ Positive } & & \multicolumn{2}{c}{ Negative } \\
\cline { 2 - 3 } & $\mathrm{n}$ & $\%$ & & $\mathrm{n}$ & $\%$ \\
\hline FISH & 85 & 28.1 & & 217 & 71.9 \\
RDT & 104 & 34.4 & & 198 & 65.6 \\
Microscopy & 74 & 24.5 & & 228 & 75.5 \\
PCR & 89 & 29.5 & & 213 & 70.5 \\
PCR \&/or Microscopy & 90 & 29.8 & & 212 & 70.2 \\
\hline
\end{tabular}

in Table 2. In the present study, Plasmodium infections diagnosed using PCR and/or microscopy were regarded as the "true positives" with the rest being regarded as "true negatives". The sensitivity and specificity of FISH was, $85.6 \%$ and $96.2 \%$ respectively, while that of microscopy was $82.2 \%$ and $100 \%$ respectively. RDT and PCR had sensitivities of, $91.1 \%$ and $98.9 \%$ respectively. The corresponding specificities were $89.6 \%$ and $100 \%$.The findings on the predictive values of the tests are shown on Table 2b. The positive predictive values (PPV) for RDT, microscopy, FISH and PCR were $78.8 \%, 100 \%, 90.6 \%$ and $100 \%$ respectively. The negative predictive values (NPV) for RDT, microscopy, FISH and PCR were, 96.0\%, 93.0\%, $94.0 \%$ and $99.5 \%$ respectively.

\section{Diagnostic effectiveness (accuracy)}

Diagnostic accuracy was expressed as a sum of the proportion of correctly diagnosed samples (true positives (TP) and true negatives (TN)) out of all the samples tested. The overall diagnostic accuracy of FISH was $93.0 \%$ while that of microscopy was $94.7 \%$. RDT and PCR had an accuracy of $90.1 \%$ and $99.7 \%$ respectively, (Table 3 ).

\section{Reliability - assessment of the agreement between the selected diagnostic approaches}

There was high level of agreement in the results of a combination of Giemsa-microscopy and PCR when assessed against FISH $(\kappa=0.803, p<0.001)$. Similarly, high levels of agreement in the results were observed when Giemsa-microscopy and PCR were assessed singly against FISH $(\kappa=0.804, p<0.001$ and $\kappa=0.822, p<0.001$, respectively). In addition, a moderate level of agreement was observed between the results obtained from RDT and those obtained by FISH $(\kappa=0.686, p<0.001)$.

\section{Malaria parasite density}

Analysis of the parasitaemia levels in the 74 samples (24.5\%) that tested positive for malaria showed that the parasite levels ranged between 173 and 229,533 parasites $/ \mu \mathrm{L}$ of blood. The geometric mean malaria parasite density was 15,631 parasites/ $\mu \mathrm{L}$ of blood. The majority of samples $(51,71.6 \%)$ had high levels of parasitaemia ( $\geq 5000$ parasites/ $\mu \mathrm{l}$ of blood). The number of cases with low (1-999 parasites/ $\mu \mathrm{L}$ of blood) and medium malaria parasite densities (1000-4999 parasites/ $\mu \mathrm{L}$ of blood) were $14(18.9 \%)$ and $7(9.5 \%)$ respectively of the samples that were positive for malaria parasites.

\section{Discussion}

The current study was undertaken to evaluate the fluorescence in situ hybridization (FISH) based technique for detecting Plasmodium sp. infection in blood smears when compared to Giemsa microscopy, PCR and RDTs. FISH is a potential test for use in malaria endemic 
Table 2 Comparison of Microscopy, FISH and RDT with Microscopy + PCR as Gold Standard

\begin{tabular}{|c|c|c|c|c|}
\hline \multirow[t]{2}{*}{ Test } & \multicolumn{4}{|c|}{$\%$ (95\% Confidence limit) } \\
\hline & Sensitivity & Specificity & Positive predictive value & Negative predictive value \\
\hline RDT & $91.1(83.4-95.4)$ & 78.8 (70.0-85.6) & $96.0(92.2-97.9)$ & $89.6(84.8-93.1)$ \\
\hline Microscopy & $82.2(73.1-88.8)$ & $100.0(95.1-100.0)$ & $93.0(88.9-95.6)$ & $100.0(98.2-100.0)$ \\
\hline $\mathrm{FISH}$ & $85.6(76.8-91.4)$ & $90.6(82.5-95.2)$ & $94.0(90.0-96.5)$ & $96.2(92.7-98.1)$ \\
\hline PCR & 98.9 (94.0-99.8) & $100.0(95.9-100.0)$ & 99.5 (97.4-99.9) & $100.0(98.2-100.0)$ \\
\hline
\end{tabular}

resource-limited settings. In particular, the use of light emitting diode (LED) as a source of light makes the technique invaluable in such settings, which characterize many sub-Saharan African countries [21]. It has been demonstrated that the LED unit with a set of blue-green filters, attached to a regular light microscope with $100 \mathrm{X}$ objective can be used to read processed FISH smears [21]. Prior to the advent of LED units, FISH processed smears were read using mercury arc lamp fluorescence microscopes [29-31]. This made the technique expensive in terms of acquisition of microscopes and also the high cost of maintenance since mercury arc lamps have a relatively short lifespan. Furthermore, the disposal of the lamps raised concerns as mercury is considered a health hazard [21]. The present LED unit with attendant light filters is relatively cheaper and offers several advantages including; (a) it is mounted onto a regular microscope, (b) it is user-friendly, (c) it can operate on a rechargeable battery unit, thus usable in remote rural areas; (d) maintenance costs are minimal since a LED unit has a lifetime of more than 10,000 h (without decaying curve); (e) the LED light source, unlike mercury bulbs, requires no focus adjustments; and (f) the LED unit does not pose any health hazard. Apart from addressing this shortcoming, the FISH assays are also able to detect as well as differentiate between the various Plasmodium species. The FISH assays detected all stages of the malaria parasites present in blood including gametocytes [21].

The potential of FISH as a vital tool for monitoring the efficacy of treatment for malaria is being considered. This is based on the fact that it detects only live parasites. FISH detects specific 18S rRNA fragments in live parasites whereas PCR, RDT and Giemsa microscopy do

Table 3 Assessment of the diagnostic accuracy of FISH, RDT, GM and PCR in malaria diagnosis against a combination PCR and GM as the gold standard

\begin{tabular}{lll}
\hline Test & Accuracy \\
\cline { 2 - 3 } & No. $(n=302)$ & $\%(95 \% \mathrm{Cl})$ \\
\hline RDT & 272 & $90.1(86.2-93.0)$ \\
Microscopy & 286 & $94.7(91.6-96.7)$ \\
FISH & 281 & $93.0(89.6-95.4)$ \\
PCR & 301 & $99.7(98.2-99.4)$ \\
\hline
\end{tabular}

not distinguish between live and dead parasites. In case of microscopy, parasite detection and speciation is based on morphology only [28], whereas FISH, in addition to speciation, based on presence of specific $18 \mathrm{~S}$ rRNA sequences, provides morphological information [21, 32].Therefore, the potential use of FISH in diagnosis, as part of the national testing algorithm, may potentially lead to the avoidance of unnecessary treatments which ultimately will enable the National Malaria Control Programme to have a better handle on the development of resistance to antimalarials [33].

In the study, it was noted that the FISH assay had the higher sensitivity (86\%) when compared to the Giemsamicroscopy (82\%) diagnostic approach which is routinely used in diagnosis centres across the country. On the other hand, it had lower sensitivity when evaluated against the RDT (91\%). As expected, PCR registered the highest sensitivity as compared to all other diagnostic approaches (99\%). The findings in this study corroborates those of a similar study by Shah and others who reported that FISH assays were more sensitive than GM. Shah and her team found that the sensitivities of P-Genus, PF and PV FISH assays to be $98.2 \%, 94.5 \%$ and $98.3 \%$, respectively compared to $89.9 \%, 83.3 \%$ and $87.9 \%$ for the detection of P. falciparum and $P$. vivax by GM respectively [21]. In the present study, 83\% (9/11) of the samples between 280 and 1000 parasites/ $\mu \mathrm{l}$ of blood and $98 \%(59 / 60)$ samples with $>2000$ parasites/ $\mu \mathrm{l}$ blood were detected by FISH. Also According to Shah et al., the limit of detection for Plasmodium Genus FISH is 170 parasites/ $\mu$ l blood [21]. Therefore, it is interesting that Osoga et al., only detected $42 \%(22 / 52)$ of the samples with $>2000$ parasites $/ \mu$ l blood and $90 \%$ of samples with 2 parasites/ $\mu$ l blood [23].

In the current study, specificities were highest in both PCR and GM (100\%), whereas RDT showed the lowest specificity (90\%). The specificity of FISH assay (96\%) was hence comparable to that of PCR and GM. Another study reported a much lower specificity for FISH assays and RDT ( $88.2 \%$ and $97.6 \%$ respectively) [21]. The discrepancies in the findings from the two studies may be attributed to, at least in part, the fact that the latter study used GM as a reference method while our study relied on PCR results in addition to Giemsa microscopy. Other studies have equally shown the superiority of FISH technique as a diagnostic tool for studying pathogens other than malaria parasites. Indeed, when 22 pathogen 
smears were tested plasmodium by FISH assays, no crossreaction was observed. The pathogens included 4 parasites (Trypanosoma cruzi, Babesia duncani, B. microti and Leishmania major), 7 bacteria and 11 viruses [34]. Similarly, Sharon and others evaluated the potential utilization of FISH for the detection and differentiation of tuberculosis from Mycobacterium tuberculosis infections in resource-constrained settings. They established that sensitivities for the Mycobacterium spp. and Mycobacterium tuberculosis FISH assay of sputum were $82 \%$ and $89 \%$; and specificities were $91 \%$ and $95 \%$, respectively [35].

In this study, the diagnostic accuracy of FISH assay was $93.0 \%$, which was higher than that of RDTs (90\%) though not statistically significant. Diagnostic accuracy of FISH assay was comparable to that of microscopy which was 95\%. Although PCR had diagnostic accuracy of about $100 \%$ its utility in malaria endemic areas is limited by the complexity of the requisite methodologies in addition to the high costs, in terms of the facilities required and the advanced level of staff training needed. Moreover, maintenance of PCR equipment is also critical, further lowering its suitability for diagnosis of malaria in remote settings and in routine clinical work [16]. On the contrary, the FISH technique is comparatively inexpensive, as it does not require high maintenance equipment. Notably, the reagents used in FISH assays are relatively thermal-stable which makes the technology useful in rural area settings.

On evaluation of the agreement between the various diagnostic approaches studied, FISH assays showed a high level of reliability in the diagnoses of malaria with the agreement with Giemsa-microscopy, and also microscopy and/or PCR being rated as high. Moderate level of agreement was found between FISH and RDT. In their research, Shah et al. also demonstrated the reproducibility of the FISH assays [21].

The present study has a number of limitations. The study did not assess the variations in the performance of the FISH assays based on the levels of parasitaemia in blood (parasite densities). Considering that FISH assays detect only live parasites while other diagnostic approaches including Giemsa microscopy detect both live and dead parasites there may be a difference in performance of the various malaria diagnostic tools [33]. The study also did not disaggregate the performance of FISH assay according to the different species of malaria parasites. However, the study provides important evidence that FISH technique may be a reasonable assay to use especially in resource-limited settings where there is a need for a sensitive, affordable and technologically sound tool for timely diagnosis of malaria.

\section{Conclusion}

This study highlights the potential use of FISH assay as an effective diagnostic tool for the detection of malaria parasites in resource-constrained settings. The specificity and reproducibility of FISH assay were found to be high. The adoption of this assay may well promote better clinical practices such as monitoring the efficacy of malaria treatment in addition to avoiding unnecessary, or minimizing empirical treatments, as it detects live parasites only. This, in turn, will lower the risk of development of drug resistance, ultimately resulting in substantial cost savings from avoidance of unwarranted treatments of patients. There remains a need to conduct further similar studies in a field setup and in different malaria epidemiological zones so as to further assess the effectiveness of this diagnostic tool. The performance of the tool also needs to be evaluated against various Plasmodium species as well as in the mixed infections cases.

\section{Abbreviations}

FISH: Fluorescence in situ hybridization; FN: False negatives; FP: False positives; GM: Giemsa microscopy; GOK: Government of Kenya; LED: Light emitting diode; MOPHS: Ministry of Public Health and Sanitation; NPV: Negative predictive value; PCR: Polymerase chain reaction; PPV: Positive predictive value; RDT: Rapid diagnostic test; TN: True negatives; TP: True positives; Unicef: United Nations Children Fund; WHO: World Health Organization

\section{Acknowledgements}

We greatly appreciate both the staff and patients at Ahero sub-County Hospital for their participation in the study.

\section{Funding}

ID-FISH Technology Inc., Palo Alto, CA, USA provided the Plasmodium FISH assay kits, for research use only. Research was supported by National Public Health Laboratories, Kenya.

\section{Availability of data and materials}

The dataset used in the current study is available from the corresponding author on reasonable request.

\section{Authors' contributions}

RK designed the study, collected data and drafted the manuscript. All the authors played participated in data analysis and interpretation of findings. Revisions of the manuscript were provided by $\mathrm{KN}$ and $\mathrm{RO}$. All authors have read and approved the final manuscript.

\section{Ethics approval and consent to participate}

Ethical review and approval of the study was obtained from the Kenyatta National Hospital/University of Nairobi Ethics and Research Committee.

Permission to carry out the study was also obtained from Ahero sub-County Hospital. The confidentiality, purpose and nature of the study were clearly explained to the participants who were requested to give written informed consent for their voluntary participation prior to the start of the study. For minors, proxy consent was obtained from their legal caretakers. Participants were free to withdraw from the study at any time without loss or penalty. All patient data collected, were kept confidentially by restricted access and coding of samples and questionnaires. Those found positive for malaria using RDT and blood slide for Giemsa staining method were treated according to the Ministry of Health guidelines.

\section{Consent for publication}

Not applicable.

\section{Competing interests}

The authors declare that they have no competing interests.

\section{Publisher's Note}

Springer Nature remains neutral with regard to jurisdictional claims in published maps and institutional affiliations. 


\section{Author details}

'Department of Medical Microbiology, College of Health Sciences, University of Nairobi, Nairobi, Kenya. ${ }^{2}$ National Malaria Control Program, Ministry of Health, Nairobi, Kenya. ${ }^{3}$ Technical University of Kenya, Nairobi, Kenya.

Received: 22 June 2017 Accepted: 30 November 2017

Published online: 08 January 2018

\section{References}

1. World Health Organization (WHO): World Malaria Report 2012. http://www.who.int/malaria/publications/world_malaria_report_2012/ en (2012). Accessed Feb 2015.

2. WHO: World Malaria Report. http://www.who.int/malaria/publications/ world_malaria_report_2014/en (2014). Accessed April 2015.

3. United Nations Children Fund (Unicef): The reality of malaria. The largest killer of children. https://www.unicef.org/health/files/health_africamalaria.pdf (2016). Accessed Feb 2015

4. WHO: Fact Sheet: World Malaria Report 2015. WHO. Geneva. http://www. who.int/malaria/publications/world-malaria-report-2016/report/en (2015). Accessed Dec 2016.

5. Amexo M, Tolhurst R, Barnish G, Bates I. Malaria misdiagnosis: effects on the poor and vulnerable. Lancet. 2004;364(9448):186-9.

6. Government of Kenya (GOK), Ministry of Public Health and Sanitation (MOPHS). National Malaria Strategy 2009-2017. Nairobi: MOPHS; 2009.

7. GOK, Ministry of Health. Kenya-Malaria- Operational-Plan. Nairobi: Government Press; 2015

8. WHO. In Kenya, the path to elimination of malaria is lined with good preventions. http://www.who.int/features/2017/vector-control-kenya/en/ (2017). Accessed Sept 2017

9. WHO: World Malaria Report 2011. http://www.who.int/malaria/world_ malaria_report_2011/en (2012). Accessed Jan 2016.

10. Joanny F, Löhr J, Engleitner T, Lell B, Mordmüller B. Limit of blank and limit of detection of plasmodium falciparum thick blood smear microscopy in a routine setting in Central Africa. Malar J. 2014;13:234.

11. Azikiwe A, Ifezulike C, Siminialayi M, Amazu U, Enye C, Nwakwunite E. A comparative laboratory diagnosis of malaria: microscopy versus rapid diagnostic test kits. Asian Pac J Trop Biomed. 2012;2:307-10.

12. Boyce MR, O'Meara WP. Use of malaria RDTs in various health contexts across sub-Saharan Africa: a systematic review. BMC public health. 2017: 18;17(1):470.

13. Maltha J, Gillet $P$, Jacobs J. Malaria rapid diagnostic tests in endemic settings. Clin Microbiol \& Infection. 2013;19:399-407.

14. Wongsrichanalai C, Barcus MJ, Muth S, Sutamihardja A, Wernsdorfer WH. A review of malaria diagnostic tools: microscopy and rapid diagnostic test (RDT). Am J Trop Med Hyg. 2007;77(6):119-27.

15. Mahende C, Ngasala B, Lusingu J, Yong TS, Lushino P, Lemnge M, Mmbando B, Premji Z. Performance of rapid diagnostic test, blood-film microscopy and PCR for the diagnosis of malaria infection among febrile children from Korogwe District, Tanzania. Malar J. 2016 26; 15(1):391.

16. Tangpukdee N, Duangdee C, Wilairatana P, Krudsood S. Malaria diagnosis: a brief review. Korean J Parasitol. 2009:47:93-102.

17. Lee C, Tzyy E, Chong J, Anderios F, Lim L, Chew H. Molecular detection of human plasmodium species in Sabah using PlasmoNexTM multiplex PCR and hydrolysis probes real-time PCR. Malar J. 2015;14:28.

18. Johnston SP, Pieniazek NJ, Xayavong MV, Slemenda SB, Wilkins PP, Da Silva AJ. PCR as a confirmatory technique for laboratory diagnosis of malaria. J Clin Microbiol. 2006;44(3):1087-9.

19. Guerin PJ, Olliaro P, Nosten F, Druilhe P, Laxminarayan R, Binka F, Kilama WL, Ford N, White J. Malaria: current status of control, diagnosis, treatment, and a proposed agenda for research and development. Lancet Infect Dis. 2002;2:564-73.

20. Kenya National Bureau of Statistics and ORC Macro. Kenya demographic and health survey 2008-09. Nairobi, Kenya and Calverton, Maryland, USA: KNBS and ORC Macro; 2010.

21. Shah J, Mark O, Weltman H, Barcelo N, Lo W, Wronska D, Kakkilaya S, Rao A, Bhat ST, Sinha R, Omar S, O'bare P, Moro M, Gilman RH, Harris N. Fluorescence in situ hybridization (FISH) assays for diagnosing malaria in endemic areas. PLoS One. 2015;10:e0136726. https://doi.org/10.1371/journal.pone.0136726.

22. Shah J, Weltman H, Narciso P, Murphy C, Poruri A, Baliga S, Sharon L, York M, Cunningham G, Miller S, Caviedes L. Dual color fluorescence in situhybridization (FISH) assays for detecting Mycobacterium tuberculosis and
Mycobacterium avium complexes and related pathogens in cultures. PLoS One. 2017:12(4):e0174989. https://doi.org/10.1371/journal.pone.0174989.

23. Osoga J, Waitumbi J, Guyah B, Sande J, Arima C, Ayaya M, Moseti C, Morang'a C, Wahome M, Achilla R, Awinda G. Comparative evaluation of fluorescent in situ hybridization and Giemsa microscopy with quantitative real-time PCR technique in detecting malaria parasites in a holoendemic region of Kenya. Malar J. 2017: 24; 16(1):297. https://doi.org/10.1186/s12936017-1943-4.

24. Lwanga SK, Lemeshow S. Determining sample size health studies: a practical manual. Geneva: WHO; 1991.

25. National Malaria Control Programme (NMCP), Kenya National Bureau of Statistics (KNBS), and ICF International. Kenya Malaria Indicator Survey 2015. Nairobi, Kenya, and Rockville, Maryland, USA: NMCP, KNBS, and ICF International; 2016.

26. WHO. Severe malaria. Trop Med Int Health. 2014. 19 (Suppl 1): 7-131. http://www.who.int/malaria/publications/atoz/who-severe-malaria-tmihsupplement-2014.pdf. Accessed Sept 2017. https://doi.org/10.1111/tmi. 12313

27. WHO: Basic Malaria Microscopy. Geneva: WHO; 1991

28. WHO: Basic Malaria Microscopy.2010. http://whqlibdoc.who.int/publications/ 2010/9789241547826 eng.pdf. Accessed Mar 2015.

29. Stender $H$, Mollerup TA, Lund K, Peterson KH, Hongmanee $\mathrm{P}$, Godtfredsen SE. Direct detection hybridization (FISH) using peptide nucleic acid (PNA) probes. Int J Tuberc Lung Dis. 1999;3(9):830-7.

30. Shah JS, Harris NS. In situ-hybridization for detecting target nucleic acid. US Patent No. 2000:6:165,723.

31. DeLong EF, Shah J. Fluorescent, ribosomal RNA probes for clinical application: a research review. Diagn Clin Testing. 1990;28:41-4.

32. DeLong EF, Wickham GS, Pace NR. Phylogenetic stains: ribosomal RNA-based probes for the identification of single cells. Science. 1989;243(4896):1360.

33. Graczyk TK, Grimes BH, Knight R, da Silva AJ, Pieniazek NJ, Veal DA. Detection of Cryptosporidium Parvum and Giardia Lamblia carried by synanthropic flies by combined fluorescent in situ hybridization and a monoclonal antibody. Am J Trop Med Hyg. 2003;68(2):228-32.

34. Shah JS, Harris NS. Detection of Babesia microti by Fluorescent In Situ-Hybridization. 39th international conference on. Antimicrob Agents Chemother. 1999:127-9.

35. Sharon L, Baliga S, Shah J, Murphy C, Weltman H. Fluorescent In Situ Hybridization (FISH) for the detection and differentiation of mycobacterium tuberculosis and NTM in sputum and culture. International Journal of Infectious Diseases. 2016;45(Suppl 1-7)

\section{Submit your next manuscript to BioMed Central and we will help you at every step:}

- We accept pre-submission inquiries

- Our selector tool helps you to find the most relevant journal

- We provide round the clock customer support

- Convenient online submission

- Thorough peer review

- Inclusion in PubMed and all major indexing services

- Maximum visibility for your research

Submit your manuscript at www.biomedcentral.com/submit 